\title{
A novel, complex RUNX2 gene mutation causes cleidocranial dysplasia
}

Wen'an $\mathrm{Xu}^{1,2+}$, Qiuyue Chen ${ }^{1,2+}$, Cuixian Liu ${ }^{3}$, Jiajing Chen ${ }^{1}$, Fu Xiong ${ }^{3^{*}}$ and Buling Wu ${ }^{1^{*}}$ (D)

\begin{abstract}
Background: Haploinsufficiency of the runt-related transcription factor 2 (RUNX2) gene is known to cause cleidocranial dysplasia (CCD). Here, we investigated a complex, heterozygous RUNX2 gene mutation in a Chinese family with CCD and the pathogenesis associated with the variations.

Methods: Genomic DNA extracted from peripheral venous blood was taken from the proband, her parents and 3 siblings, and 150 normal controls. Analysis of their respective RUNX2 gene sequences was performed by PCR amplification and Sanger sequencing. Pathogenesis associated with RUNX2 mutations was investigated by performing bioinformatics, real-time PCR, western blot analysis, and subcellular localization studies.

Results: We identified 2 complex heterozygous mutations involving a c.398-399 insACAGCAGCAGCAGCA insertion and a c.411-412 insG frameshift mutation in exon 3 of the RUNX2 gene. The frameshift mutation changed the structure of the RUNX2 protein while did not affect its expression at the mRNA level. Transfection of HEK293T cells with a plasmid expressing the RUNX2 variant decreased the molecular weight of the variant RUNX2 protein, compared with that of the wild-type protein. Subcellular localization assays showed both nuclear and cytoplasmic localization for the mutant protein, while the wild-type protein localized to the nucleus.

Conclusions: Our findings demonstrated that the novel c.398-399insACAGCAGCAGCAGCA mutation occurred alongside the c.411-412insG frameshift mutation, which resulted in RUNX2 truncation. RUNX2 haploinsufficiency was associated with CCD pathogenesis. These results extend the known mutational spectrum of the RUNX2 gene and suggest a functional role of the novel mutation in CCD pathogenesis.
\end{abstract}

Keywords: Craniofacial anomalies, Oral systemic disease(s), RUNX2, Molecular genetics, Haploinsufficiency, Truncation protein

\section{Background}

Cleidocranial dysplasia (CCD, OMIM 119600) is an autosomal dominant human skeletal disorder resulting from haploinsufficiency of the Runt-related transcription factor 2 (RUNX2) gene, a master regulator for bone and cartilage development and maintenance [1-6]. CCD is characterized by a myriad of skeletal abnormalities and short stature. Skeletal abnormalities associated with CCD include hypoplastic or aplastic clavicles, patent

\footnotetext{
* Correspondence: xiongfu@smu.edu.cn; bulingwu@smu.edu.cn

${ }^{\dagger}$ Equal contributors

${ }^{3}$ Department of Medical Genetics, School of Basic Medical Sciences, Southern Medical University, Guangzhou, Guangdong, China

'Department of Stomatology, Nanfang Hospital, College of Stomatology,

Southern Medical University, Guangzhou, Guangdong, China

Full list of author information is available at the end of the article
}

sutures and fontanelles, dental abnormalities, and other skeletal abnormalities $[2,7,8]$.

RUNX2 haploinsufficiency causes CCD and, although most CCD patients have a family history of CCD, approximately one-third of CCD patients were found to lack RUNX2 mutations [4, 9]. Here, we investigated a Chinese patient with CCD and identified 2 complex heterozygous RUNX2 mutations. To investigate the function and potential pathogenic mechanism of the RUNX2 mutant, we performed bioinformatics, real-time PCR, western blot analysis, and subcellular localization studies. Our results suggested that the novel mutations changed the molecular weight, structure, nuclear localization, and expression of the RUNX2 protein. 


\section{Methods}

\section{Patients}

The proband (patient II-1), a 17-year-old girl, was referred to the Department of Stomatology at Nanfang Hospital for consultation regarding a dental abnormality. An experienced pediatric dentist performed clinical examinations for the proband and her family. Medical histories were obtained from the family members, including her parents and 3 siblings. One hundred and fifty normal controls from healthy individuals matched for gender and ethnic origin were recruited from Nanfang Hospital in Guangzhou, Guangdong. All subjects gave informed consent and the study was approved by the Ethics Committee of Southern Medical University.

\section{RUNX2-gene mutation screening}

To identify disease-associated mutations, we extracted genomic DNA from the peripheral blood of the proband and her family members by a standard phenol/chloroform extraction method. PCR reactions were performed using primers designed with Primer3 plus software, the sequences of which are shown in Table 1. The PCR products were visualized by $1.5 \%$ agarose gel electrophoresis and subsequently analyzed by Sanger sequencing.

To identify RUNX2 (NCBI Reference Sequence: NM_001015051.3) gene mutations, PCR products corresponding to exon 3 of RUNX2 were cloned into the PMD-18 T vector (TaKaRa Biotechnology, Dalian, Co., Ltd) and introduced into DH5 $\alpha$ bacteria (TaKaRa Biotechnology). Transformants were then isolated, and the RUNX2 gene sequences were studied by PCR and DNA sequencing.

\section{RNA analysis}

Total RNA was extracted from the peripheral blood of the proband and her parents with TRIzol (Invitrogen, Carlsbad, CA, USA) and purified by chloroform extraction and isopropanol precipitation. Total RNA samples were quantified by measuring the absorbance at 260 and

Table 1 Primers used in RUNX2polymerase chain reaction (PCR)

\begin{tabular}{lll}
\hline Positions & $\begin{array}{l}\text { Forward nucleotide sequence } \\
\left(5^{\prime}-3^{\prime}\right)\end{array}$ & $\begin{array}{l}\text { Reverse nucleotide sequence } \\
\left(5^{\prime}-3^{\prime}\right)\end{array}$ \\
\hline Exon 1 & AGAGAGAGAAAGAGCAAGGGG & GCATAGACTGTGGTAGAGAGC \\
Exon 2 & TाTCTTTGCTTTCACATGTTACC & TGCTATTTGGAAAAGTAGCAG \\
Exon 3 & CGCTAACTTGTGGCTGTTG & CGTGGGCAGGAAGACACC \\
Exon 4 & CATTCCTGTCGGCCATTACTG & CATCAAAGGAGCCTAATGTGCT \\
Exon 5 & AAGTGGTCATCGGAGGGTT & TGCAGATAGCAAAGTCCACAA \\
Exon 6 & GGCCACCAGATACCGCTTAT & CCAGCGTCTATGCAAGTGAA \\
Exon 7 & GCCTGAAAGGATGGGGTTAT & CTGTGCAGGGATGGATTIT \\
Exon 8 & CTTATGGGCCTGCAGACTCT & AGTAACAACCAGACAGCCCA \\
Exon 9 & CTGTGGCTTGCTGTTCCTTT & TGATACGTGTGGGATGTGGC \\
\hline
\end{tabular}

$280 \mathrm{~nm}$. Reverse transcriptase-polymerase chain reactions (RT-PCR) were performed using the PrimeScript RT-PCR Kit (TaKaRa Biotechnology). Quantitative RTPCR (qRT-PCR) was performed to compare peripheral blood RUNX2 mRNA expression levels between the patient and her parents. qRT-PCR was performed using Platinum SYBR Green (Bio-Rad Laboratories, California, USA) and an MxPro Real-Time PCR System (Stratagene MX3005P), using 40 cycles of $95{ }^{\circ} \mathrm{C}$ for $20 \mathrm{~s}, 63{ }^{\circ} \mathrm{C}$ for $20 \mathrm{~s}$, and $72{ }^{\circ} \mathrm{C}$ for $20 \mathrm{~s}$. The sequences of the primers used for the qRT-PCR experiments are shown in Table 2. Each sample was analyzed in triplicate, and $\beta$-actin mRNA expression was measured as a reference. Student's 2 -tailed $t$-test was used for statistical analysis.

\section{Bioinformatics}

Three-dimensional structures of the wild-type and mutant RUNX2 protein were predicted using the I-TASSER server $[10,11]$.

\section{Construction of recombinant plasmids}

PCR fragments encoding the mutant or wild-type RUNX2 gene were amplified using primers designed by Oligo 7 (sequences shown in Table 3) to have an annealing temperature of $63{ }^{\circ} \mathrm{C}$. The PCR products were extracted from a $1.5 \%$ agarose gel using the SanPrep Column DNA Gel Extraction Kit (Sangon Biotech, Shanghai). The purified PCR product for the mutant gene was then cloned into the PMD-20 $\mathrm{T}$ vector (TaKaRa Biotechnology) to generate the PMD-20 TRUNX2 mutant construct. After sequence confirmation, the inserted mutant RUNX2 gene was then subcloned into the pEGFP-C1 vector via the SalI and XamI restriction sites, generating a recombinant plasmid (pEGFPC1-RUNX2) encoding the mutant RUNX2 gene. A recombinant plasmid encoding the wild-type RUNX2 gene was constructed using the same method.

\section{Western blot analysis}

To study protein expression, recombinant plasmids encoding wild-type or mutant RUNX2 templates, as well as the parental vector ( $\mathrm{pEGFP-C1)}$, were transfected separately into human embryonic kidney (HEK) 293 T cells. After $24 \mathrm{~h}$ in culture, total proteins were extracted with cell lysis buffer for western blotting (Beyotime, Shanghai) containing of Protease Inhibitor Cocktail (Sigma, St. Louis, MO). The proteins were resolved by sodium dodecyl sulfate-polyacrylamide gel electrophoresis. The separated proteins were transferred to polyvinylidene

Table 2 Primers used for qRT-PCR

Positions Forward nucleotide sequence Reverse nucleotide sequence $\left(5^{\prime}-3^{\prime}\right) \quad\left(5^{\prime}-3^{\prime}\right)$

q-RUNX2 TCCTCCCCAAGTAGCTACCT GAGGCGGTCAGAGAACAAAC 
Table 3 Primers designed by oligo 7

\begin{tabular}{lll}
\hline Name & $\begin{array}{l}\text { Forward nucleotide sequence } \\
\left(5^{\prime}-3^{\prime}\right)\end{array}$ & $\begin{array}{l}\text { Reverse nucleotide sequence } \\
\left(5^{\prime}-3^{\prime}\right)\end{array}$ \\
\hline RUNX2 & ATGGCATCAAACAGCCTCTTC & TCAATATGGTCGCCAAACAGA
\end{tabular}

difluoride membranes (Millipore). After the membranes were blocked in TBST containing 5\% non-fat milk, they were incubated overnight with a primary antibody against enhanced green fluorescence protein (Santa Cruz Biotechnology, USA). Next, the membranes were incubated for $2 \mathrm{~h}$ with an appropriate secondary antibody (Santa Cruz Biotechnology) at room temperature. Relative RUNX2 protein expression levels were calculated after normalization to glyceraldehyde 3-phosphate dehydrogenase (Santa Cruz Biotechnology) expression. Protein expression was detected using the Immobilon Western Chemiluminescent HRP Substrate (Thermo Fisher, USA).

\section{Cell localization studies}

Recombinant plasmids encoding wild-type or mutant RUNX2 genes were transfected into COS7 cells to study the subcellular localization of the RUNX2 protein and the impact of the mutation. At $48 \mathrm{~h}$ post-transfection, cells were rinsed 3 times with PBS, and nuclei were stained with $0.1 \mu \mathrm{g} / \mathrm{ml} \mathrm{4',6-diamidino-2-phenylindole}$ (Sigma) for $10 \mathrm{~min}$ at room temperature. Subsequently, the cells were viewed under an Eclipse Ti-U fluorescence microscope (Nikon; Tokyo, Japan).

\section{Results}

\section{Clinical features of the proband patient}

The patient underwent a detailed clinical evaluation by an experienced dentist. Physical examination revealed short stature (height: $143 \mathrm{~cm}$ ), dental abnormalities, a high-arched palate, open fontanelles, and ptotic and hypermobile shoulders. The remaining clinical features tested for were normal. The family history and physical examinations revealed no other family members with bony abnormalities.

Detailed dental abnormalities, including impacted supernumerary teeth, retention of primary teeth, eruption failure of permanent teeth, and abnormal root development were very instructive for devising a treatment plan (Fig. 1a, b). Delayed closure of the cranial sutures and detailed dental abnormalities were identified by computed tomography (Fig. 1c, d). Chest radiographs showed hypoplastic or aplastic clavicles and structural abnormalities of the right shoulderpeak joint (Fig. 1e). The overall characteristics of the clinical and radiographic results supported a clinical diagnosis of CCD.

\section{Sequence analysis of RUNX2 gene}

All 9 coding exons of the RUNX2 gene were amplified by PCR. Direct screening revealed complex mutations in exon 3 of the patient's gene, which were not identified in the parents' DNA (Fig. 2b). Thus, these mutations arose de novo, as neither parent carried these mutations, and non-paternity was excluded by paternity testing.

BLAST analysis revealed novel mutations (c.398-399 insACAGCAGCAGC AGCA and c.411-412insG) in cis in the runt domain of the patient's RUNX2 gene (Fig. 2c, d). The insertions created a frameshift mutation that led to the translated nornal proteins changing into truncated proteins containing 165 amino proteins. Both mutations were absent in DNA samples from 150 unrelated, normal control subjects that were matched for Chinese ethnicity to rule out the possibility of these mutations occurring naturally as single-nucleotide polymorphisms.

\section{Functional analysis}

Our qRT-PCR results showed that the patient's RUNX2 messenger RNA expression level was not significantly different from those of her parents (Fig. 3a). However, western blot analysis revealed that transfection with a wild-type RUNX2 construct resulted in the production of a $56-\mathrm{kDa}$ protein, whereas transfection with the mutant RUNX2 construct produced an $18-\mathrm{kDa}$, truncated protein (Fig. 3b). These molecular masses matched the expected sizes of the encoded RUNX2 variants. Threedimensional structures predicted with the I-TASSER server showed the anticipated structural changes resulting from the RUNX2, exon 3 mutations (Fig. 3d).

The exon 3 mutations affected subcellular location of the RUNX2 variant. The mutant protein expressed from the pEGFP-C1-RUNX2 plasmid was distributed throughout both the cytoplasm and nucleus of transfected COS7 cells, whereas the wild-type protein was localized only in the nucleus (Fig. 3c).

\section{Discussion}

Mutations in the RUNX2 gene, that maps to the short arm of chromosome 6, cause CCD [12]. The RUNX2 gene encodes a transcription factor that is a member of the core-binding factor family. In this report, we present data from a patient with a novel in-frame insertion mutation in the RUNX2 gene and CCD symptoms, such as disproportionate short stature and the classic triad of multiple supernumerary teeth, open sagittal sutures and fontanelles, and hypoplastic or aplastic clavicles [13, 14]. Apart from these typical clinical features, CCD patients can show other symptoms, such as mental deficiencies, hearing disorders, median pseudo-cleft palates, delayed ossification of the pelvis, and other skeletal abnormalities [15-17]. CCD is an autosomal dominant genetic disease; thus, patient's parents generally harbor the same 

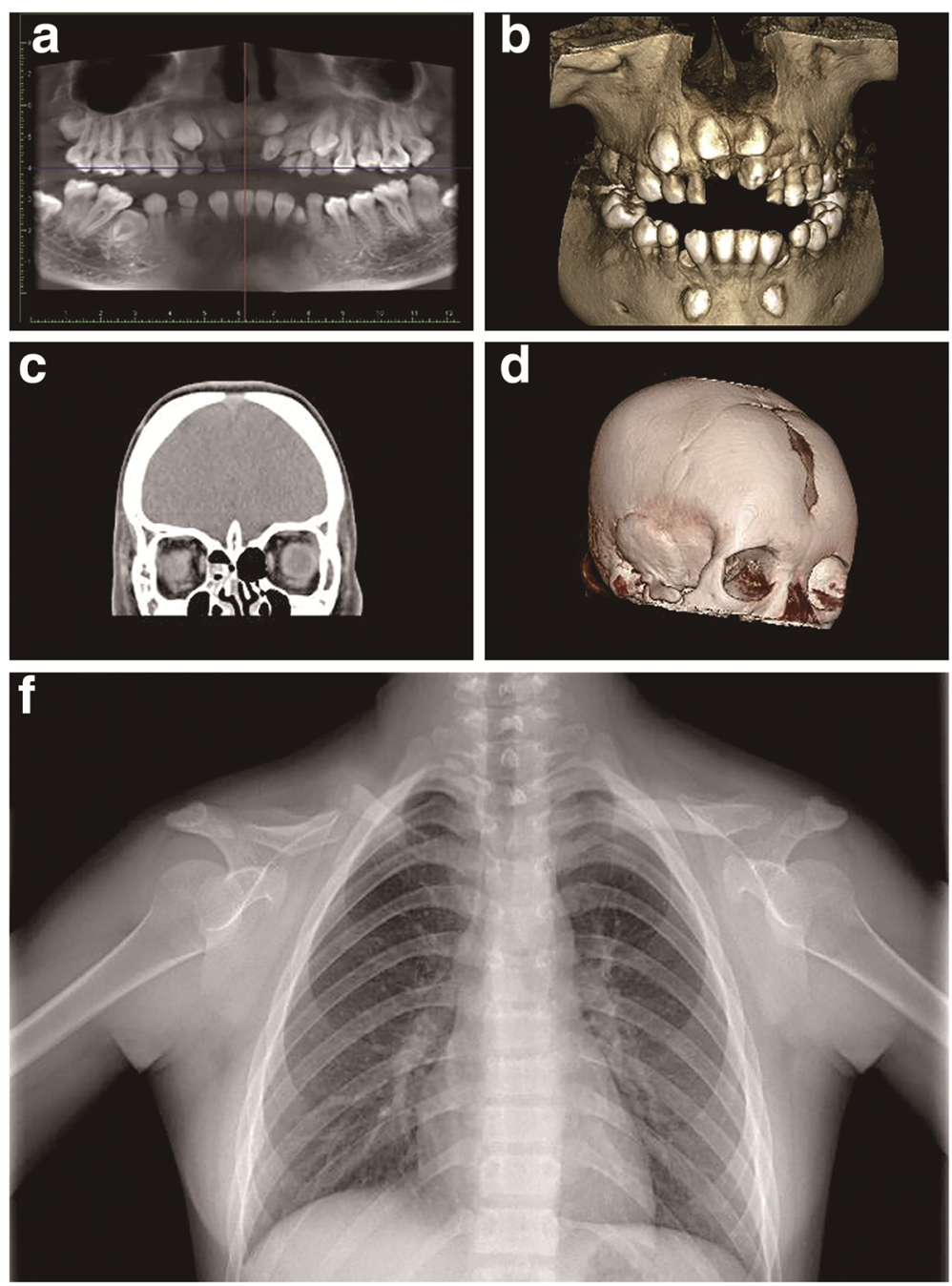

Fig. 1 Radiological findings for the patient. a, b Cone-beam computed tomography results showing detailed dental abnormalities, including impacted supernumerary teeth, the retention of primary teeth, eruption failure of the permanent teeth, and impaired root development. c, d A skull CT scan showed the presence of open fontanelles. e Radiographs revealed hypoplastic or aplastic distal ends of clavicles and structural abnormalities occurring in the right shoulder peak joint

genetic mutation, although patients with unaffected parents have been reported in several studies $[4,9,13]$. In this study, insertional mutations were found in exon 3 of the patient's RUNX2 gene, while her parents lacked these mutations. Paternity testing excluded the nonpaternity between the proband and her parents, thereby indicating that the genetic abnormalities arose as de novo events. We propose that the de novo mutations should have arisen during the spermatogenic process or an early stage in embryonic development.

Although CCD-related bone anomalies develop through an unclear mechanism(s), it is known that CCD results from RUNX2-dependent signaling pathways. The RUNX2 protein regulates extracellular matrix properties and mineralization through transforming growth factor$\beta$-responsive, COL10A1, VEGF, and MMP13 pathways, providing supporting evidence that RUNX2 affects endochondral ossification, intramembranous ossification, and chondrocyte maturation $[16,18,19]$. The RUNX2 protein forms a complex with core-binding factor $\beta(\mathrm{CBF} \beta)$ and binds to a conserved nucleotide sequence (R/ TACCRCA) to drive expression of several osteogenic proteins, such as collagen a1, osteopontin, bone sialoprotein, and osteocalcin [20,21]. Collectively, these findings indicate that RUNX2 plays an important role in osteoblast differentiation. Periodontal ligament stem cells modulate root resorption in human primary teeth via the RUNX2-regulating, receptor activator of nuclear factor kappa-B ligand pathway, causing the retention of primary teeth [22]. The RUNX2 protein can be thought of as having 4 domains, consisting of the $\mathrm{N}$ terminus, the runt domain, the PST domain, and the $\mathrm{C}$ terminus. 
a

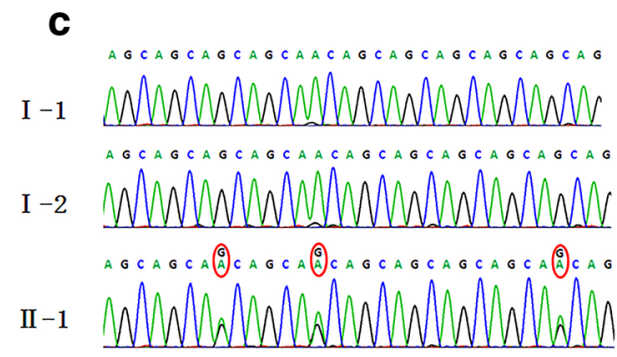

b

WT
MUT
II

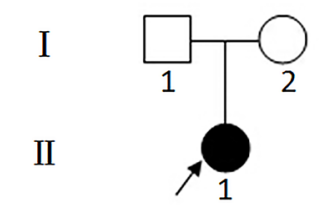

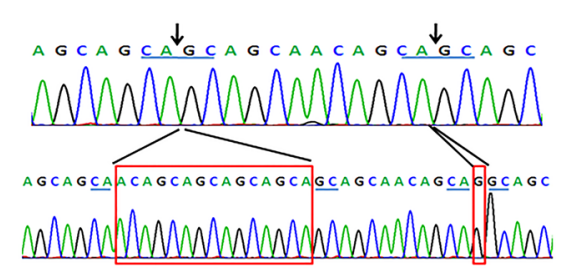

d

TG C T G C T G C T G C T G T T G C

MWWWWWW

tactactectactattac

MWMWWWW

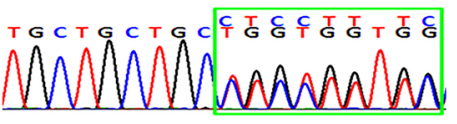

Fig. 2 Mutation analysis. a Family pedigree. The arrow indicates the proband in the family. b Mutation screening. Sequences of the wild-type (WT) and mutant (MUT) RUNX2 gene sequences occurring in exon 3. Nucleotides highlighted in the red boxes show the insertion mutations. c Forward DNA sequencing results. Panels I-1 and I-2 show the sequences of wild-type RUNX2 alleles, and panel II-1 shows double peaks within this region. $\mathbf{d}$ Reverse DNA sequencing results. Panels $1-1$ and $1-2$ show sequences of WT RUNX2 alleles, and panel II-1 shows a double-peak phenomenon
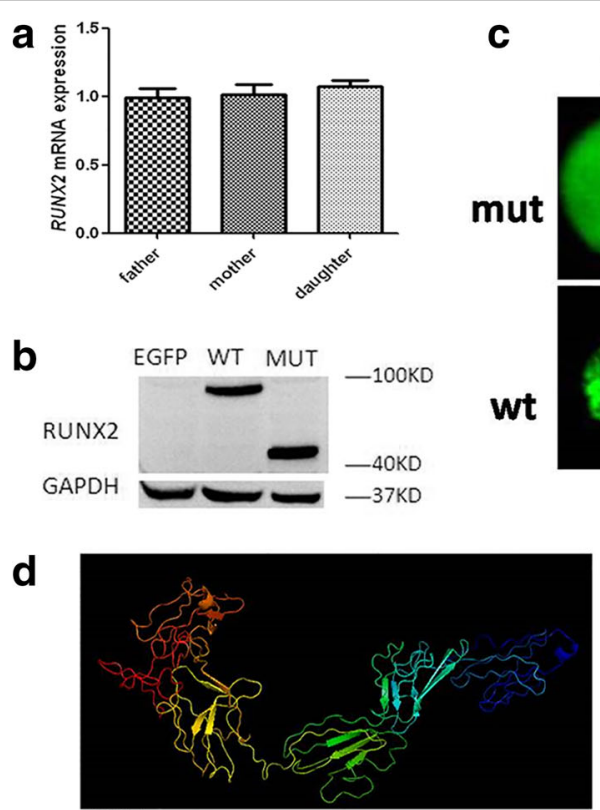

Wild-type
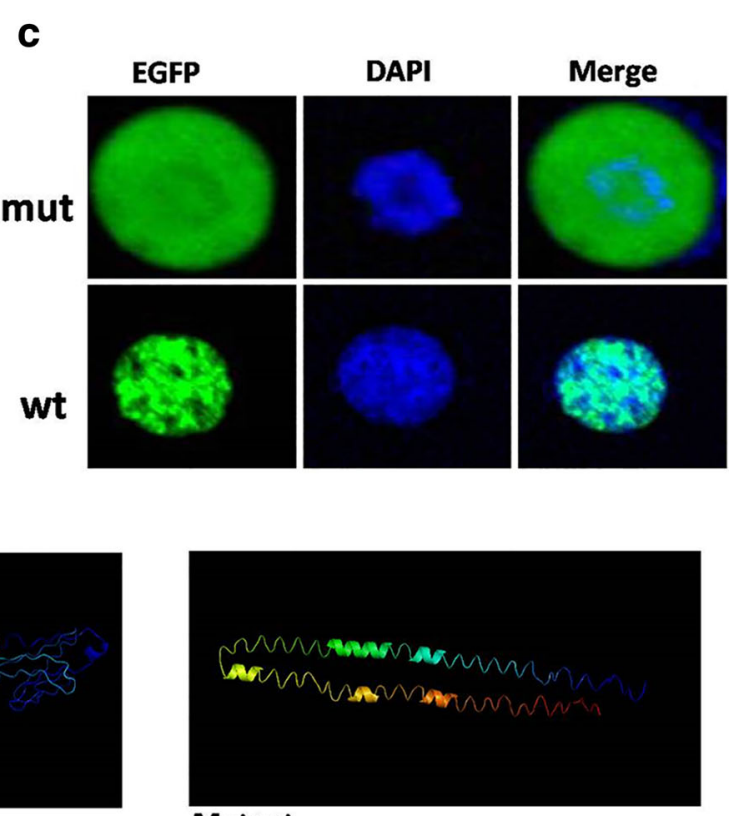

Mutant

Fig. 3 Functional analysis of wild-type and mutant RUNX2. a Quantification of RUNX2 mRNA expression levels revealed no significant difference $(P=0.6218)$ between the patient and her parents. $\mathbf{b}$ Western blot analysis of RUNX2 protein expression showed that transfection of the wild-type RUNX2 construct resulted in full-length RUNX2 protein production, whereas overexpression of mutant RUNX2 generated a truncated protein. GAPDH, $37 \mathrm{kDa}$. c COS7 cells were transfected with recombinant plasmids encoding the wild-type or mutant RUNX2 genes. Confocal micrographs showed the intracellular distributions of the wild-type and mutant RUNX2 proteins. $\mathbf{d}$ Molecular modeling performed using the I-TASSER server revealed that the mutant runt domain plays a critical role in the normal 3-dimensional structure of RUNX2, as indicated 
The runt domain and nuclear localization signal (NLS) at the C-terminal domain border is important for the transcriptional activity, subcellular distribution, and aggregation of the RUNX2 protein [23-25]. Furthermore, many findings have revealed that the runt domain is responsible for DNA binding and heterodimerization with the $C B F \beta$ protein $[3,4]$ The $C B F \beta$ protein is an unrelated binding partner that enhances the DNA-binding affinity of the RUNX2 protein [26].

In the present study, the patient showed normal RUNX2 mRNA expression compared with her parents, which indicated that the mutation did not affect mRNA transcription. However, the insertional mutation occurring in the patient led to premature translation termination, producing a truncated protein containing 165 amino proteins. Structural modeling suggested that the normal molecular structure of the truncated protein was altered, thereby abolishing the function of the runt domain, which could also redirect nuclear RUNX2 from the nucleus to the cytoplasm to prevent binding to $\mathrm{CBF} \beta$ $[24,25]$. In addition, the truncated protein lost the NLS, which is normally present at the C-terminal border. This alteration could impair the transcriptional activation function of RUNX2, resulting in decreased ossification and skeletal deformities $[1,23]$.

\section{Conclusions}

Haploinsufficiency of the RUNX2 gene is known to be the reason of CCD. Here, we present a molecular cytogenetic characterization of novel RUNX2 insertional mutations occurring in a 17-year-old female patient with $\mathrm{CCD}$ and discuss potential genotype-phenotype correlations in this case. Data presented here expand the known RUNX2 mutation spectrum and potentially shed insight into the development of CCD.

\section{Abbreviations \\ CBFß: Core-binding factor $\beta$; CCD: Cleidocranial dysplasia; HEK: Human embryonic kidney; NLS: Nuclear localization signal; qRT-PCR: Quantitative RT-PCR; RT-PCR: Reverse transcriptase-polymerase chain reactions; RUNX2: Runt-related transcription factor 2}

\section{Acknowledgements}

We thank all participants in this study for their cooperation.

\section{Funding}

This work was supported by grants from the National Natural Science Foundation of China (No. 81371137; No. 31371279) and the Natural Science Foundation of Guangdong (No. S2012010009551).

\section{Availability of data and materials}

The datasets supporting the conclusions of this article are included within the article.

\section{Authors' contributions}

BW, FX conceived and designed the experiments. WX, QC, CL, and JC prepared the manuscript and performed the experiments. QC, $C L$ analyzed and interpreted the data. FX, BW, WX and QC were responsible for critical revision of the content. All authors read and approved the final manuscript.

\section{Competing interests}

The authors declare that they have no competing interests.

\section{Consent for publication}

Consent for publication was obtained from all subjects involved in this study.

\section{Ethics approval and consent to participate}

Study approval was obtained from the Southern Medical University Ethics Committee. Detailed written informed consent was obtained from all participants or their guardians.

\section{Author details}

'Department of Stomatology, Nanfang Hospital, College of Stomatology, Southern Medical University, Guangzhou, Guangdong, China. ${ }^{2}$ Department of Stomatology, Zhongshan City People's Hospital, Zhongshan, Guangdong, China. ${ }^{3}$ Department of Medical Genetics, School of Basic Medical Sciences, Southern Medical University, Guangzhou, Guangdong, China.

Received: 22 June 2016 Accepted: 26 January 2017

Published online: 07 February 2017

\section{References}

1. Lee B, Thirunavukkarasu K, Zhou L, Pastore L, Baldini A, Hecht J, et al. Missense mutations abolishing DNA binding of the osteoblast-specific transcription factor OSF2/CBFA1 in cleidocranial dysplasia. Nat Genet. 1997; 16:307-10.

2. Matheny CJ, Speck ME, Cushing PR, Zhou Y, Corpora T, Regan M, et al. Disease mutations in RUNX1 and RUNX2 create nonfunctional, dominantnegative, or hypomorphic alleles. EMBO J. 2007;26:1163-75.

3. Mundlos S, Otto F, Mundlos C, Mulliken JB, Aylsworth AS, Albright S, et al. Mutations involving the transcription factor CBFA1 cause cleidocranial dysplasia. Cell. 1997:89:773-9.

4. Jensen BL, Kreiborg S. Development of the dentition in cleidocranial dysplasia. J Oral Pathol Med. 1990;19:89-93.

5. Tang $S, X u$ Q, Xu X, Du J, Yang X, Jiang Y, et al. A novel RUNX2 missense mutation predicted to disrupt DNA binding causes cleidocranial dysplasia in a large Chinese family with hyperplastic nails. BMC Med Genet. 2007;8:82.

6. Chen W, Ma J, Zhu G, Jules J, Wu M, McConnell M, Tian F, Paulson C, Zhou $X$, Wang $L$, et al. Cbfbeta deletion in mice recapitulates cleidocranial dysplasia and reveals multiple functions of Cbfbeta required for skeletal development. Proc Natl Acad Sci U S A. 2014;111(23):8482-7.

7. Bergwitz C, Prochnau A, Mayr B, Kramer FJ, Rittierodt M, Berten HL, et al. Identification of novel CBFA1/RUNX2 mutations causing cleidocranial dysplasia. J Inherit Metab Dis. 2001;24:648-56.

8. Yoshida T, Kanegane H, Osato M, Yanagida M, Miyawaki T, Ito Y, et al. Functional analysis of RUNX2 mutations in Japanese patients with cleidocranial dysplasia demonstrates novel genotype-phenotype correlations. Am J Hum Genet. 2002;71:724-38.

9. Feldman GJ, Robin NH, Brueton LA, Robertson E, Thompson EM, SiegelBartelt J, et al. A gene for cleidocranial dysplasia maps to the short arm of chromosome 6. Am J Hum Genet. 1995;56:938-43.

10. Yang J, Yan R, Roy A, Xu D, Poisson J, Zhang Y. The I-TASSER Suite: protein structure and function prediction. Nat Methods. 2015;12:7-8.

11. Roy A, Kucukural A, Zhang Y. I-TASSER: a unified platform for automated protein structure and function prediction. Nat Protoc. 2010;5(4):725-38.

12. Quack I, Vonderstrass B, Stock M, Aylsworth AS, Becker A, Brueton L, et al. Mutation analysis of core binding factor $A 1$ in patients with cleidocranial dysplasia. Am J Hum Genet. 1999:65:1268-78.

13. Suda N, Hattori M, Kosaki K, Banshodani A, Kozai K, Tanimoto K, et al. Correlation between genotype and supernumerary tooth formation in cleidocranial dysplasia. Orthod Craniofac Res. 2010;13:197-202.

14. Wu LZ, Su WQ, Liu YF, Ge X, Zhang Y, Wang XJ. Role of the RUNX2 p.R2250 mutation in cleidocranial dysplasia: a rare presentation and an analysis of the RUNX2 protein structure. Genet Mol Res. 2014;13:1187-94.

15. Chang JL, Brauer DS, Johnson J, Chen CG, Akil O, Balooch G, et al. Tissuespecific calibration of extracellular matrix material properties by transforming growth factor-beta and Runx2 in bone is required for hearing. EMBO Rep. 2010;11:765-71.

16. Takenouchi T, Sato W, Torii C, Kosaki K. Progressive cognitive decline in an adult patient with cleidocranial dysplasia. Eur J Med Genet. 2014;57:319-21. 
17. Zheng Q, Sebald E, Zhou G, Chen Y, Wilcox W, Lee B, et al. Dysregulation of chondrogenesis in human cleidocranial dysplasia. Am J Hum Genet. 2005; 77:305-12.

18. Lu Y, Li Y, Cavender AC, Wang S, Mansukhani A, D'Souza RN. Molecular studies on the roles of Runx2 and Twist1 in regulating FGF signaling. Dev Dyn. 2012;241:1708-15.

19. Ducy P, Zhang R, Geoffroy V, Ridall AL, Karsenty G. Osf2/Cbfa1: a transcriptional activator of osteoblast differentiation. Cell. 1997:89:747-54.

20. Komori T. Regulation of bone development and extracellular matrix protein genes by RUNX2. Cell Tissue Res. 2010;339:189-95.

21. Li B, Zhang Y, Wang $Q$, Dong Z, Shang L, Wu L, et al. Periodontal ligament stem cells modulate root resorption of human primary teeth via Runx2 regulating RANKL/OPG system. Stem Cells Dev. 2014;23:2524-34.

22. Zaidi SK, Javed A, Choi JY, van Wijnen AJ, Stein JL, Lian JB, et al. A specific targeting signal directs Run×2/Cbfa1 to subnuclear domains and contributes to transactivation of the osteocalcin gene. J Cell Sci. 2001;114:3093-102.

23. Kanno T, Kanno Y, Chen LF, Ogawa E, Kim WY, Ito Y. Intrinsic transcriptional activation-inhibition domains of the polyomavirus enhancer binding protein 2/core binding factor alpha subunit revealed in the presence of the beta subunit. Mol Cell Biol. 1998;18:2444-54.

24. Pelassa I, Cora D, Cesano F, Monje FJ, Montarolo PG, Fiumara F. Association of polyalanine and polyglutamine coiled coils mediates expansion diseaserelated protein aggregation and dysfunction. Hum Mol Genet. 2014;23: 3402-20.

25. Qin X, Jiang Q, Matsuo Y, Kawane T, Komori H, Moriishi T, et al. Cbfb regulates bone development by stabilizing runx family proteins. J Bone Miner Res. 2015;30:706-14.

26. Bartfeld D, Shimon L, Couture GC, Rabinovich D, Frolow F, Levanon D, et al. DNA recognition by the RUNX1 transcription factor is mediated by an allosteric transition in the RUNT domain and by DNA bending. Structure. 2002;10:1395-407.

\section{Submit your next manuscript to BioMed Central and we will help you at every step:}

- We accept pre-submission inquiries

- Our selector tool helps you to find the most relevant journal

- We provide round the clock customer support

- Convenient online submission

- Thorough peer review

- Inclusion in PubMed and all major indexing services

- Maximum visibility for your research

Submit your manuscript at www.biomedcentral.com/submit

) Biomed Central 